\title{
Protection of Deep Neural Networks for Face Recognition with Adversarial Attacks
}

\author{
Alice Guo \\ Morgantown High School, 109 Wilson Ave, Morgantown, WV 26501, USA; aliceguo168@gmail.com
}

ABSTRACT: Artificial Intelligence (AI) is a transformative technology with the potential to revolutionize our entire lives. The discovery of deep neural networks (DNNs), which give computers the ability to outperform humans in many things, including face recognition, was a milestone that has made AI so inviting. However, scientists have discovered very recently that DNN networks are especially vulnerable to attacks, i.e., the so-called adversarial examples, which are imperceptible to humans but can fool DNNs easily. In this project, a novel approach is proposed to defend against these adversaries, which is much more efficient than the often-used defense methods based on adversarial learning. The newly proposed idea utilizes the attacks themselves as a defense mechanism. The task of face recognition is used to experimentally validate the novel idea and approaches. Based on a large dataset with 6,000 pairs of face images, this new defense handles the adversarial attacks efficiently, improving the face recognition accuracies from about $0 \%$ under attack to over $80 \%$ after defense.

KEYWORDS: Deep learning; Deep Neural Networks; Adversarial attacks; Defense; Face recognition.

\section{- Introduction}

The field of artificial intelligence is essentially when machines do tasks that typically require human intelligence. It encompasses machine learning, where machines can learn through experience. Deep learning ${ }^{1,2}$ is a subset of machine learning where artificial neural networks, inspired by the human brain, learn from large amounts of data. Deep learning is referred to as such because the neural networks have various (deep) layers that enable learning. The application of deep learning has affected almost every aspect of our daily lives. Deep Neural Networks (DNNs) have become the preferred choice as a means of solving many challenging tasks in face recognition, image classification, speech recognition, and natural language processing. ${ }^{1}$

Recent advances in deep learning allow neural networks to achieve the state-of-the-art results for most machine learning tasks. However, it has been shown that neural networks are vulnerable to adversarial examples. ${ }^{3,6}$ It is easy to modify inputs so that they indistinguishable from the original data for humans, and yet classified incorrectly by the network. Figure 1 shows an example of an adversarial attack where the image of a panda has been misclassified as a gibbon. Adversarial attacks such as shown in Figure 1 make it difficult to apply deep learning models in security-critical environments. Because of this, adversarial attacks and their defenses have drawn increasing attention in recent years.

Most research focusing on adversarial attacks and defenses involve developing algorithms for the problem of image classification, based on the datasets, e.g., MNIST, CIFAR-10, CIFAR-100, ImageNet, and developed algorithms, e.g., Fast Gradient Sign Method (FGSM), ${ }^{3}$ or C\&W's attack, ${ }^{5}$ usually do not consider the specific image content, like a human face or a special object. On the other hand, human face recognition (FR) is a different problem with many practical applications. Deep networks developed for face recognition could be attacked as well. Currently, there are a small number of approaches to attacking face recognition systems, which are specially designed for perturbation of face images to attack FR systems, ${ }^{7,9}$ by modifying facial features and/or by facial landmark manipulations. Thus, a question is raised: is the development of special attacks against face images needed in order to attack face recognition systems? In other words, are the attacks developed for image classification applicable to effectively attack face recognition?

It is unclear whether the attacks developed for image classification apply to face images without detecting any facial content or details. A knowledge gap exists, which is one of the motivations for this work. The purpose of this project is to examine whether the attack methods take effect on face images without searching any facial content or details. If the answer is yes, researchers should make efforts to deal with these general attacks for face recognition systems; in contrast, if the answer is no, there should be less concern about face recognition attacks, and the focus should be on developing special attacks to face images by utilizing the facial features or landmarks in order to attack the FR systems.

Therefore, it was of great importance to investigate the representative attacks developed for image classification for their effect on face recognition. Additionally, a new scheme based on positively utilizing the attacks themselves is developed to protect deep networks for face recognition under attack.

The main contributions of this work include:

- An investigation and selection of the representative adversarial attack methods originally developed for image classification, but applied to a different problem domain- face recognition; 
- An exploration of the selected attack methods' effect and performance on face recognition;

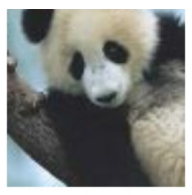

$x$

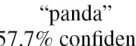

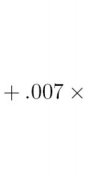

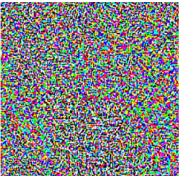

$\operatorname{sign}\left(\nabla_{x} J(\boldsymbol{\theta}, \boldsymbol{x}, y)\right)$

"nematode"

$8.2 \%$ confidence

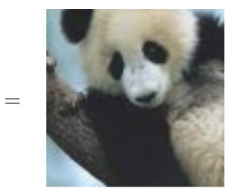

$x+$

$\epsilon \operatorname{sign}\left(\nabla_{\boldsymbol{x}} J(\boldsymbol{\theta}, \boldsymbol{x}, y)\right)$ "gibbon" $99.3 \%$ confidence

Figure 1: An adversarial image generated by Fast Gradient Sign Method: ${ }^{3}$ left: a clean image of panda; middle: the adversarial perturbation; right: adversarial image, classified as a gibbon.

- A proposed and validated new scheme based on a smart and positive use of the attacks to make face recognition systems more robust against adversarial attacks.

In the following paragraphs, Section 2 presents some representative methods for adversarial attacks in image classification. Section 3 describes a new investigation of the attacks on face recognition and proposes a new scheme for defense. Experimental validations are conducted in Section 4. Finally, some conclusions are given.

\section{Methods for Adversarial Attacks}

Quite a few methods have been developed to perform adversarial attacks to deep networks ${ }^{10}$ with typical attack applications on image classification. After careful examination of various attack methods, three were selected for the investigation on face recognition, and brief descriptions of the selected methods are presented here, followed by a short overview of defense approaches.

Fast Gradient Sign Method (FGSM):

Goodfellow et al. proposed a method called Fast Gradient Sign Method (FGSM), ${ }^{3}$ which performs one-step gradient updates along the sign direction of the gradient at each pixel. The algorithm can be expressed as:

$$
x^{a d v}=x+\epsilon \cdot \operatorname{sign}\left(\nabla_{x} L(x, y, \theta)\right),
$$

where $\mathcal{E}$ is the magnitude of the perturbation and $L(x, y$, $\theta$ ) is the original loss function (such as the cross-entropy loss for image classification). $x$ is the clean image, $y$ is the ground truth label, $\theta$ is the set of parameters, and $x^{a d v}$ is the adversarial example after the attack.

FGSM updates each pixel location of image $x$ by a magnitude of $\varepsilon$ following the direction that increases the original loss function. Targeted attack towards a given label $l$ is also easy to perform, only requiring a flipped sign in Eq. (1) and changing the ground truth label $y$ to the targeted label $l$.

\section{Projected Gradient Descent (PGD:}

A more powerful adversary is the multi-step variant FGSMk, which is essentially projected gradient descent (PGD) on the negative loss function: ${ }^{4}$

$$
x_{k+1}^{a d v}=x_{k}^{a d v}+\alpha \cdot \operatorname{sign}\left(\nabla_{x_{k}^{a d v}} L\left(x_{k}^{a d v}, y, \theta\right)\right) \text {, }
$$

where the PGD first finds the adversarial example by iteratively adding the gradient sign with the previous adversarial example in Eq. (2). $\alpha$ in the equation is a small constant. Also, small, random noise is added to the clean image before the first iteration, which may slightly improve the attack success rate.

One main difference between PGD and many other adversarial methods is that PGD only uses adversarial data to update the parameters of the model, rather than using both the clean and adversarial data as in many other methods.

CE'W's Attack (CEW:

The C\&W's Attack $(\mathrm{CW})^{5}$ is with a new adversarial loss function,

$$
\begin{gathered}
\min _{\delta}\|\delta\|_{p}+c \cdot g(x+\delta) \\
\text { s.t. } x+\delta \in[0,1]^{d}
\end{gathered}
$$

where $g\left(x^{a d v}\right) \geq 0$ if and only if $f\left(x^{a d v}\right)=l$. Here $l$ is the targetted attack label, $\delta$ is the pixel perturbation, $p$ is the norm control, $c$ is a constant, and $d$ is the image size.

The distance along the penalty term can be easily optimized with any gradient-based method (FGSM, PGD, etc.). Several different objective functions can be used for $g(x)$. One of the most commonly used is:

$$
g\left(x^{a d v}\right)=\max \left(\max _{i \neq l}\left(f_{i}\left(x^{a d v}\right)\right)-f_{l}\left(x^{a d v}\right),-k\right)
$$

Where $f(x)$ denotes the output from Softmax function. This objective pushes the Softmax score for targeted attack class $l$ to be higher than all other classes $i$ by a margin $k$.

\section{Adversarial Defenses:}

Adversarial defense mainly follows two approaches. These methods differ in the stage where defense is applied: either at the data input stage or at the model stage. Currently, the most effective defense methods are model stage defenses. An example of this is adversarial training, ${ }^{4,11}$ where models are trained with adversarial examples, and thus, become more robust to adversarial attack during the test time. In practice, however, adversarial training can take a very long time, e.g. days to learn with a robust model.

Unlike model stage defense, input stage defense does not make any changes to the original model. Such defense methods either detect for an adversarial example before feeding it into the model, ${ }^{12,13}$ or reconstruct the input data so that the adversarial perturbation is removed. ${ }^{14,15}$

One major concern with input stage defense is that the defense accuracy tends to drop significantly when the attacker knows the detection or reconstruction pipeline and modifies the attack accordingly. ${ }^{10}$ It is also quite hard to remove the perturbations and recover the clean images from the attacked adversarial examples.

\section{New Attacks}

So far, there are only a small number of approaches related to attack and defense for face recognition. Existing approaches ${ }^{7,9}$ are specially designed for attacking face images, using facial properties or landmarks, while a number of methods were developed for attack and defense to image classification. It was worth investigating a whether the attack and defense methods developed for natural image classification can be applied to face recognition or not since face recognition is considered a different task from image classification., 


\section{Attacks to Face Recognition:}

After a careful examination of the attack methods, three representative methods were selected among those attacks, the FGSM, ${ }^{3} \mathrm{PGD},{ }^{4}$ and $\mathrm{C} \& \mathrm{~W},{ }^{5}$ as presented in the previous section, applying to attacking face recognition. Reasons for selecting these three representatives are: (1) The FGSM is a classical one-step attack method, which can be computed effectively based on the gradient operation; (2) The PGD and $\mathrm{C} \& \mathrm{~W}$ are two effective iterative methods with multiple iterations in attacking image classification systems. The three selected examples are sufficiently representative for attacks to image classification.

The benefits of examining the image classification attacks on face recognition include: (1) If the attacks developed for image classification can be applied to face recognition effectively, one may not need to pursue the use of special properties from facial regions in designing the face attack algorithms; Instead, one may just treat the face images as some "general" images; (2) On the other hand, if the attacks designed for image classification have no effect on face recognition, one may not need to worry about those attacks in developing a face recognition system. Therefore, it is of great importance to investigate the effect on face recognition on the attacks designed to attack image classification systems.

\section{Defenses for Face Recognition:}

In addition to applying the attacks on face recognition and investigating the attack effects, it is also useful to develop an approach to defend the attacks on face recognition.

A novel defense idea is presented here utilizes the attacks to change the clean face images of the adversarial images, and then the face matching is changed to measure the similarity between two adversarial face images, rather than between one clean face image and one attacked image.

By applying the adversarial attack to the query face image and gallery database face image, both images are converted into adversarial images, and the problem is reformulated as matching between two adversarial images. In this way, it not only avoids the need to retrain the face recognition model with adversarial examples, which could take days or weeks even for a relatively small dataset, ${ }^{4}$ but also circumvents the unnecessary trade-off between robustness and model accuracy, as in previous approaches. ${ }^{16}$ So, there are some useful properties for the proposed new approach as compared to adversarial training and other complex defense methods developed so far.

\section{Results and Discussion}

The datasets and the deep networks used for face recognition are introduced first. Then the validation of the selected attacks on face recognition are presented. Next, the new scheme for defense is evaluated.

\section{Dataset and Deep Networks:}

Web-collected data sets were used, including CASIA-WebFace with 453,453 images over 10,575 identities (a cleaned version of the original dataset), and LFW with 6000 pairs of face images, including 3000 pairs of the same person (positive pairs) and 3000 pairs of different people (negative pairs). In the experiments, CASIA-WebFace was used to train the deep pairs) and 3000 pairs of different people (negative pairs). In the experiments, CASIA-WebFace was used to train the deep model for face recognition, and then the LFW was used to evaluate the trained model for face verification.

All the faces in images were aligned by MTCNN algorithm ${ }^{17}$ and cropped to $160 \times 160$ RGB images for FaceNet ${ }^{18}$ and $112 \times 112$ for CosFace. ${ }^{19}$ Following convention, each pixel (in $[0,255]$ ) in RGB images was normalized by subtracting 127.5 and then dividing by 128 in testing. For training, random flipping and per-data whitening was used.

The network used was the same as the used in FaceNet + CosFace with the SphereFace Network. ${ }^{20}$ The pre-trained model was provided by the inventors, which was trained on CASIA-WebFace and tested on LFW. For face verification, an input image was fed to a pre-trained model, and the extracted feature of that image was a size of 512 for FaceNet and 1024 for CosFace. Then, the distance, or similarity, was computed for every pair of images. As a result, the verification accuracy was $98.9 \%$ for FaceNet and $99.3 \%$ for CosFace, showing that the face models can work very well for face recognition without attacks. For the following experiments, only the CosFace model was used, because of its higher accuracy.

\section{Attacks on Face Recognition:}

The most commonly used adversarial attack methods, FGSM, PGD, and C\&W attacks, originally proposed for image classification tasks, were selected for examining their effect on face recognition.

In the experimental validation, all perturbations were added onto the face images in LFW dataset for the face verification task. For experimental results, the accuracies of verification were compared between two cases: before attacking and after attacking, to evaluate the attack methods' capability to attack face recognition. For all attacks, the perturbations of images were constrained within the pixel value difference of 2, 4, and 8 (for the 0 to 255 range). FGSM is a one-step attack, which means the perturbation is executed only once. However, the PGD and C\&W are iterative; thus, the perturbation calculations are done many times. In the end, the accuracies for face verification with adversarial attacks on the LFW are shown in Table 1.

From Table 1, it is clear that the three selected attack methods can disrupt the face recognition system significantly. For instance, even the one-step attack method FGSM can reduce the face recognition accuracy from $99.3 \%$ to $7.5 \%$ when the perturbation range is within 4 pixel values, and can be reduced further to $2.9 \%$ when the perturbation is allowed to have 8 pixel values. The iterative methods PGD and C\&W can be more effective than the FGSM in attacking. For example, the face recognition accuracy can be reduced from $99.3 \%$ to $0.0 \%$ when the perturbations are within 4 pixel values. Based on these experimental results, one can see clearly that the attack methods originally developed for image classification can attack the face recognition systems significantly without identifying any special facial properties or manipulating any facial landmarks. So, based on the results here, effective attack methods can be develop for face recognition systems without utilizing the facial fiducial points or any other special features 
or properties in those methods to attack face recognition.

Table 1: Face verification accuracy after attacks. The separate accuracies for positive and negative pairs are reported, in addition to the accuracy for all pairs. Some key parameter settings are reported as well.

\begin{tabular}{|l|l|l|l|l|}
\hline $\begin{array}{l}\text { Attack } \\
\text { Method }\end{array}$ & & Acc.(\%) & $\begin{array}{l}\text { Acc.(\%) } \\
\text { (pos.) }\end{array}$ & $\begin{array}{l}\text { Acc.(\%) } \\
\text { (neg.) }\end{array}$ \\
\hline \multirow{3}{*}{ FGSM } & eps=2.0 & 30.20 & 30.80 & 29.50 \\
\cline { 2 - 5 } & eps=4.0 & 7.50 & 7.30 & 7.70 \\
\hline \multirow{3}{*}{ eps=8.0 (Iter 10) } & 2.90 & 1.80 & 3.90 \\
\hline \multirow{3}{*}{ C\&W (Iter 10) } & $\begin{array}{l}\text { step size=0.5 } \\
\text { eps=2.0 }\end{array}$ & 3.40 & 3.50 & 3.30 \\
\cline { 2 - 5 } & $\begin{array}{l}\text { step size=1.0 } \\
\text { eps=4.0 }\end{array}$ & 0.00 & 0.00 & 0.00 \\
\cline { 2 - 5 } & $\begin{array}{l}\text { step size=2.0 } \\
\text { eps=8.0 }\end{array}$ & 0.00 & 0.00 & 0.00 \\
\hline & $\begin{array}{l}\text { step size=0.5 } \\
\text { eps=2.0 }\end{array}$ & 3.40 & 3.50 & 3.30 \\
\cline { 2 - 5 } & $\begin{array}{l}\text { step size=1.0 }=4.0 \\
\text { eps }\end{array}$ & 0.00 & 0.00 & 0.00 \\
\hline & $\begin{array}{llll}\text { step size=2.0 } \\
\text { eps=8.0 }\end{array}$ & 0.00 & 0.00 & 0.00 \\
\hline
\end{tabular}

\section{Defenses for Face Recognition Systems:}

After showing the effectiveness of the selected attack methods on face recognition, a further question arises: how do we develop effective defense methods to deal with those attacks and make the face recognition systems more robust?

In the field of image classification, the most commonly used defense methods are based on the adversarial training ${ }^{3,21}$ by adding the adversarial examples with the clean images in the training stage. However, adversarial training can be very time consuming, may take days or weeks even on a relatively small dataset, ${ }^{4}$ and also has to trade-off between robustness and model accuracy. ${ }^{16}$

Table 2: Face verification accuracy after using PGD defense.

\begin{tabular}{|c|c|c|c|c|c|c|c|c|c|}
\hline & \multicolumn{3}{|c|}{$\begin{array}{l}\text { num_step: } 10 \\
\text { step_ize: } 2.0\end{array}$} & \multicolumn{3}{|c|}{$\begin{array}{l}\text { num_step: } 20 \\
\text { step_size: } 1.0\end{array}$} & \multicolumn{3}{|c|}{$\begin{array}{l}\text { numstep: } 40 \\
\text { step_size: } 0.5\end{array}$} \\
\hline & \begin{tabular}{|l}
$\begin{array}{l}\text { Acc. (\%) } \\
\text { overall }\end{array}$ \\
\end{tabular} & $\begin{array}{l}\text { Acc. (\%) } \\
\text { (pos.) }\end{array}$ & $\begin{array}{l}\begin{array}{l}\text { Acc. (\%) } \\
\text { (neg.) }\end{array}\end{array}$ & \begin{tabular}{|l}
$\begin{array}{l}\text { Acc. (\%) } \\
\text { overall }\end{array}$ \\
\end{tabular} & $\begin{array}{l}\text { Acc. (\%) } \\
\text { (pos.) }\end{array}$ & $\begin{array}{l}\text { Acc. (\%) } \\
\text { (neg.) }\end{array}$ & \begin{tabular}{|l|}
$\begin{array}{l}\text { Acc. (\%) } \\
\text { overall }\end{array}$ \\
\end{tabular} & $\begin{array}{l}\text { Acc. (\%) } \\
\text { (pos.) }\end{array}$ & $\begin{array}{l}\text { Acc. (\%) } \\
\text { (neg.) }\end{array}$ \\
\hline \begin{tabular}{|l} 
PGD vs PGD \\
\end{tabular} & 2.70 & 1.00 & 4.40 & 37.9 & 34.2 & 41.7 & 71.4 & 65.9 & 76.9 \\
\hline \begin{tabular}{|l|} 
FGSM+PGD vs PGD \\
\end{tabular} & 26.0 & 16.9 & 35.1 & 58.8 & 0.5 & 67.2 & 80.6 & 74.7 & 86.6 \\
\hline \begin{tabular}{|l|} 
PGD + PGD vs PGD \\
\end{tabular} & 45.2 & 37.7 & 52.6 & 73.8 & 68.7 & 78.9 & 88.0 & 84.5 & 91.5 \\
\hline \begin{tabular}{|l}
$\mathrm{C} \& W+\mathrm{PGD}$ vs $\mathrm{PGD}$ \\
\end{tabular} & $45 \cdot 2$ & 37.5 & 52.8 & 74.1 & 69.0 & 79.2 & 87.8 & 84.4 & $\begin{array}{l}91.2 \\
\end{array}$ \\
\hline
\end{tabular}

Table 3: Face verification accuracy after using C\&W defense.

\begin{tabular}{|c|c|c|c|c|c|c|c|c|c|}
\hline & \multicolumn{3}{|c|}{$\begin{array}{l}\text { num_step: } 10 \\
\text { step_size: } 20\end{array}$} & \multicolumn{3}{|c|}{$\begin{array}{l}\text { num_ttep: } 20 \\
\text { stepsize: } 1.0\end{array}$} & \multicolumn{3}{|c|}{$\begin{array}{l}\text { num_step: } 40 \\
\text { step_size: } 0.5\end{array}$} \\
\hline & 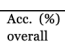 & $\begin{array}{l}\text { stepsize: } 2 \\
\text { Acc. (\%) } \\
\text { (pos.) }\end{array}$ & $\begin{array}{l}\begin{array}{l}\text { Acc. (\%) } \\
\text { (neg.) }\end{array}\end{array}$ & \begin{tabular}{|l}
$\begin{array}{l}\text { Acc. (\%) } \\
\text { overall }\end{array}$ \\
\end{tabular} & $\begin{array}{l}\text { steps.size: } \\
\text { Acc. } \\
\text { (pos.) }\end{array}$ & $\begin{array}{l}\text { Acc. (\%) } \\
\text { (neg.) }\end{array}$ & \begin{tabular}{|l}
$\begin{array}{l}\text { Acc. (\%) } \\
\text { overall }\end{array}$ \\
\end{tabular} & $\begin{array}{l}\text { steps.ize: } \\
\text { Acc. (\%) } \\
\text { (pos.) }\end{array}$ & 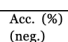 \\
\hline \begin{tabular}{|l}
$\mathrm{C} \& \mathrm{~W}$ vs $\mathrm{C} \& \mathrm{~W}$ \\
\end{tabular} & 13.5 & 11.4 & 11.6 & 43.4 & 38.1 & 48.2 & 72.1 & 66.4 & 77.8 \\
\hline FGSM $+\mathrm{C} \& W$ vs $\mathrm{C} \& \mathrm{~W}$ & 26.0 & & 35.2 & 59.0 & 49.9 & \begin{tabular}{|l|l|}
68.1 \\
\end{tabular} & 80.8 & 74.3 & 87.3 \\
\hline \begin{tabular}{|l}
$\mathrm{PGD}+\mathrm{C} \& \mathrm{~W}$ vs C\&W \\
\end{tabular} & 45.4 & \begin{tabular}{|l|}
37.7 \\
\end{tabular} & 53.0 & 74.4 & \begin{tabular}{|l|l|}
69.9 \\
\end{tabular} & \begin{tabular}{|c|}
79.0 \\
\end{tabular} & 88.1 & \begin{tabular}{|l|l|}
84.7 \\
\end{tabular} & 91.6 \\
\hline $\begin{array}{l}\mathrm{C} \& \mathrm{~W}+\mathrm{C} \& \mathrm{~W} \text { vs } \mathrm{C} \& \mathrm{~W} \\
\end{array}$ & $\begin{array}{l}\frac{45.4}{45.1} \\
4\end{array}$ & \begin{tabular}{|l|l|}
37.2 \\
37.2
\end{tabular} & $\frac{53.0}{52.9}$ & \begin{tabular}{|l}
74.4 \\
7.4 \\
\end{tabular} & \begin{tabular}{|l|l|}
69.3 \\
\end{tabular} & \begin{tabular}{|l|l|}
779.4 \\
\end{tabular} & 87.9 & \begin{tabular}{|l|l|}
84.1 \\
4.6 \\
\end{tabular} & \begin{tabular}{|l|l|l|l}
91.2 \\
\end{tabular} \\
\hline
\end{tabular}

In this exploration to develop a defense for face recognition systems, the author takes a unique approach to using the attacks to create a novel and effective defense. The key idea is to apply the attack to clean face images before matching in such a way that the face matching is between two attacked face images rather than between an attacked image and a clean one.

The defense results are shown in Tables 2, 3, and 4. In each table, the results are shown for face matching of "Query" vs "Gallery", which indicates what attack methods were applied to the query face image and gallery image, respectively. For example, the first row in Table 2: "PGD vs PGD", means that the PGD attack was applied to the query face image and gallery face image, individually. In the first row of each of the three tables, the results were to show the case when the same attack methods are applied to the query and gallery face images separately. That is, assuming that the attack methods were known (to the query), and the same attack methods were applied to the gallery face images as well. In this case, both the PGD and C\&W methods did well for defense. For instance, after 40 iterations, the face recognition accuracies can be raised from $0 \%$ to $71.4 \%$ for the PGD method and to $72.1 \%$ for the C\&W method. However, as shown in Table 4, the FGSM method did not raise the face recognition accuracy. So, to use the attacks to perform defense, the FGSM attack did not work while the PGD and C\&W methods improved the face recognition accuracies significantly.

In practice, the attacks may not be known to the defense system. To perform the defense for unknown attacks, the three attack methods were validated with a different setting. No matter what the attack method was on the query face image, a specified attack method (one of the FGSM, PGD, and $\mathrm{C} \& W$ ) was applied to the query image again, and the same attack method was applied to the gallery face image as well.

The experimental results using this approach are shown in Tables 2, 3 and 4 in rows 2-4. For example, in Table 2 row 2: the FGSM attack was applied to the query face image which was unknown to the defense system. The PGD attack method was the specified method by the defense system, so the PGD attack was applied to the query face image (already attacked by the FGSM), and also applied to the gallery face image. The face recognition accuracy was raised from $0 \%$ to $80.6 \%$ by using the PGD attack as the specified defense and the recognition accuracy was improved significantly.

Table 4: Face verification accuracy after using FGSM defense.

\begin{tabular}{|c|c|c|c|c|c|c|c|c|c|}
\hline & \multicolumn{3}{|c|}{$\begin{array}{l}\text { num_step: } 10 \\
\text { ste__size: } 2.0\end{array}$} & \multicolumn{3}{|c|}{ num_step: 20} & \multicolumn{3}{|c|}{$\begin{array}{l}\text { num_tep: } 40 \\
\text { step_size: } 0.5\end{array}$} \\
\hline & \begin{tabular}{|l}
$\begin{array}{l}\text { Acc. (\%) } \\
\text { overall }\end{array}$ \\
\end{tabular} & $\begin{array}{l}\text { Actes. (19te) } \\
\text { (pos.) }\end{array}$ & $\begin{array}{l}\text { Acc. }(\%) \\
\text { (neg.) }\end{array}$ & $\begin{array}{l}\begin{array}{l}\text { Acc. (\%) } \\
\text { overall }\end{array} \\
\end{array}$ & 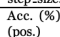 & $\begin{array}{l}\text { Acc. (\%) } \\
\text { (neg.) }\end{array}$ & \begin{tabular}{|l|}
$\begin{array}{l}\text { Acc. (\%) } \\
\text { overall }\end{array}$ \\
\end{tabular} & $\begin{array}{l}\text { Acc-size. } \\
\text { (pos). }\end{array}$ & 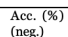 \\
\hline \begin{tabular}{|l} 
FGSM vs FGSM \\
\end{tabular} & \begin{tabular}{|l|l|l|l|l|l|l}
6.20 \\
\end{tabular} & \begin{tabular}{|l} 
(pos.) \\
12.2 \\
\end{tabular} & \begin{tabular}{|l|l|} 
(neg. \\
0.20
\end{tabular} & $\begin{array}{l}\text { Overail } \\
0.10 \\
\end{array}$ & \begin{tabular}{|l|l|} 
(pos. \\
0.20 \\
\end{tabular} & $\begin{array}{l}\text { (neg.) } \\
0.00\end{array}$ & \begin{tabular}{|l|l} 
overall \\
.00 \\
\end{tabular} & \begin{tabular}{|l|l|} 
poss. \\
0.00 \\
\end{tabular} & \\
\hline FGSM+FGSM vs FGSM & 0.00 & \begin{tabular}{|l|l|}
0.00 \\
\end{tabular} & \begin{tabular}{|l|l|}
0.00 \\
\end{tabular} & \begin{tabular}{|l|l|}
0.00 \\
\end{tabular} & 0.00 & 0.00 & 0.00 & 0.00 & 0.00 \\
\hline $\begin{array}{l}\text { PGD+FGSM vs FGSM } \\
\end{array}$ & \begin{tabular}{|l|l|}
0.00 \\
\end{tabular} & \begin{tabular}{|l|l|}
0.00 \\
\end{tabular} & 0.00 & $\begin{array}{ll}0.00 \\
\end{array}$ & 0.00 & 0.00 & 0.00 & 0.00 & $\begin{array}{ll}0.00 \\
\end{array}$ \\
\hline
\end{tabular}

Similarly, a check of other rows in the three tables, shows that even when the attacks were unknown to the defense system, the PGD and C\&W methods (Tables 2 and 3) still worked well for defense. All recognition accuracies were raised above $80 \%$ by both methods. However, the FGSM method did not work, since almost all accuracies were about 0\%, as shown in Table 4.

To summarize the experimental results, both the PGD and C\&W attack methods work quite well in serving as a defense, whether the attacks to the query face images are known or unknown. The FGSM attack does not work for any defense.

\section{- Conclusion}

Adversarial attacks on face recognition were investigated with three representative attacks, the FGSM, PGD, and C\&W, selected to perform attacks on face recognition. The validations revealed that the three attacks can make face recognition performance worse, reducing the recognition accuracies from about $99 \%$ to $0 \%$. This result is of great importance, showing that the design of attacks to face recognition systems does not need to use special facial properties or landmarks, which were utilized previously to attack face recognition. Furthermore, a novel use of the attacks was proposed and performed for defense. Experimental results show that the PGD and C\&W attacks can be used to perform defense effectively, raising face reecognition accuracies from $0 \%$ to around $80 \%$ or above, regardless of whether the attacks are known or unknown; this is of great significance in practice, since the attacks can be used positively for defense result. Future research could show deeper insights into the mechanism of attacks and defense, and validate future attacks developed for defense purposes. 


\section{Acknowledgements}

The author thanks X. Xu and C. Chen for some help on coding and running experiments and reviewers for previewing the paper.

\section{References}

1. Yann LeCun, Yoshua Bengio, and Geoffrey Hinton. Deep learning. nature, 521(7553):436, 2015.

2. Ian Goodfellow, Yoshua Bengio, and Aaron Courville. Deep learning. MIT press, 2016.

3. Ian J Goodfellow, Jonathon Shlens, and Christian Szegedy. Explaining and harnessing adversarial examples. arXiv preprint arXiv:1412.6572, 2014.

4. Aleksander Madry, Aleksandar Makelov, Ludwig Schmidt, Dimitris Tsipras, and Adrian Vladu. To- wards deep learning models resistant to adversarial attacks. arXiv preprint arXiv:1706.06083, 2017..

5. Nicholas Carlini and David Wagner. Towards evaluating the robustness of neural networks. In Symposium on Security and Privacy, pages 39-57. IEEE, 2017

6. Nicolas Papernot, Patrick McDaniel, Ian Goodfel- low, Somesh Jha, Z Berkay Celik, and Ananthram Swami. Practical blackbox attacks against ma- chine learning. In Proceedings of the 2017 ACM on Asia Conf. on Computer and Communications Security, pages 506-519. ACM, 2017.

7. Qing Song, Yingqi Wu, and Lu Yang. Attacks on state-of-the-art face recognition using attentional adversarial attack generative network. arXiv preprint arXiv:1811.12026, 2018.

8. Mahmood Sharif, Sruti Bhagavatula, Lujo Bauer, and Michael K Reiter. Adversarial generative nets: Neural network attacks on state-of-the-art face recognition. arXiv preprint arXiv:1801.00349, 2017.

9. Ali Dabouei, Sobhan Soleymani, Jeremy Dawson, and Nasser M Nasrabadi. Fast geometrically- perturbed adversarial faces. arXiv preprint arXiv:1809.08999, 2018.

10.Anish Athalye, Nicholas Carlini, and David Wagner. Obfuscated gradients give a false sense of security: Circumventing defenses to adversarial examples. arXiv preprint arXiv:1802.00420, 2018.

11.Florian Tram`er, Alexey Kurakin, Nicolas Paper- not, Ian Goodfellow, Dan Boneh, and Patrick Mc- Daniel. Ensemble adversarial training: Attacks and defenses. arXiv preprint arXiv:1705.07204, 2017.

12.Jan Hendrik Metzen, Tim Genewein, Volker Fischer, and Bastian Bischoff. On detecting adversarial perturbations. arXiv preprint arXiv:1702.04267, 2017.

13.Reuben Feinman, Ryan R Curtin, Saurabh Shintre, and Andrew B Gardner. Detecting adversarial samples from artifacts. arXiv preprint arXiv:1703.00410, 2017

14.Arjun Nitin Bhagoji, Daniel Cullina, and Prateek Mittal. Dimensionality reduction as a defense against evasion attacks on machine learning classifiers. arXiv preprint arXiv:1704.02654, 2017.

15.Dongyu Meng and Hao Chen. Magnet: a two- pronged defense against adversarial examples. In Proceedings of the 2017 ACM SIGSAC Conference on Computer and Communications Security, pages 135-147. ACM, 2017.

16.Dimitris Tsipras, Shibani Santurkar, Logan Engstrom, Alexander Turner, and Aleksander Madry. Robustness may be at odds with accuracy. stat, 1050:11, 2018.

17.K. Zhang, Z. Zhang, Z. Li, and Y. Qiao. Joint face detection and alignment using multitask cascaded convolutional networks. IEEE Signal Processing Letters, 23(10):1499-1503, 2016.

18.Florian Schroff, Dmitry Kalenichenko, and James Philbin. Facenet: A unified embedding for face recognition and clustering. In Proceedings of the IEEE conference on computer vision and pattern recognition, pages 815-823, 2015.

19.Hao Wang, Yitong Wang, Zheng Zhou, Xing Ji, Dihong Gong, Jingchao Zhou, Zhifeng Li, and Wei Liu. Cosface: Large margin cosine loss for deep face recognition. In Proceedings of the IEEE Conference on Computer Vision and Pattern Recognition, pages 5265-5274, 2018.

20.Weiyang Liu, Yandong Wen, Zhiding Yu, Ming Li, Bhiksha Raj, and Le Song. Sphereface: Deep hypersphere embedding for face recognition. In Proceedings of the IEEE conference on computer vision and pattern recognition, pages 212-220, 2017.

21.Ruitong Huang, Bing Xu, Dale Schuurmans, and Csaba Szepesv'ari. Learning with a strong adversary. arXiv preprint arXiv:1511.03034, 2015.

\section{Author}

Alice Guo is currently a senior at Morgantown High School in Morgantown, WV, but completed this research project as a freshman. She is the recipient of research awards at the international level and has been recognized by organizations and government agencies for her work. With strong interests in computer programming, artificial intelligence, mathematics, and writing, she plans to major in Computer Science in college. 